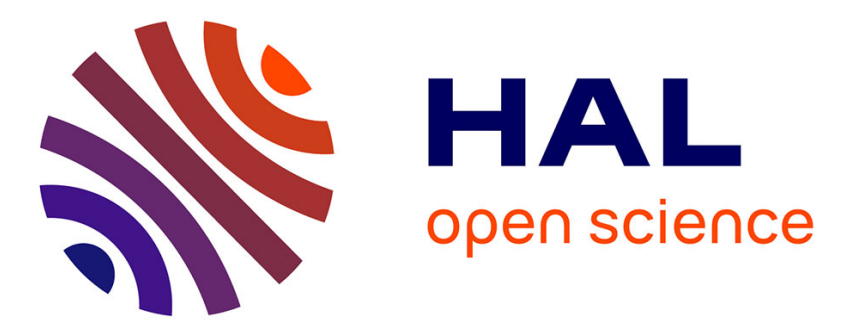

\title{
ÉTUDE PAR DIFFRACTION BRILLOUIN DES DOMAINES ACOUSTOÉLECTRIQUES DANS LE SULFURE DE CADMIUM
}

J. Attal, Pascal Girard, M. Rouzeyre

\section{- To cite this version:}

J. Attal, Pascal Girard, M. Rouzeyre. ÉTUDE PAR DIFFRACTION BRILLOUIN DES DOMAINES ACOUSTOÉLECTRIQUES DANS LE SULFURE DE CADMIUM. Journal de Physique Colloques, 1972, 33 (C6), pp.C6-156-C6-159. 10.1051/jphyscol:1972635 . jpa-00215153

HAL Id: jpa-00215153

https://hal.science/jpa-00215153

Submitted on 1 Jan 1972

HAL is a multi-disciplinary open access archive for the deposit and dissemination of scientific research documents, whether they are published or not. The documents may come from teaching and research institutions in France or abroad, or from public or private research centers.
L'archive ouverte pluridisciplinaire HAL, est destinée au dépôt et à la diffusion de documents scientifiques de niveau recherche, publiés ou non, émanant des établissements d'enseignement et de recherche français ou étrangers, des laboratoires publics ou privés. 


\title{
ÉTUDE PAR DIFFRACTION BRILLOUIN DES DOMAINES ACOUSTOÉLECTRIQUES DANS LE SULFURE DE CADMIUM
}

\author{
J. ATTAL, P. GIRARD et M. ROUZEYRE
}

Université des Sciences et Techniques du Languedoc, place E. Bataillon, 34000 Montpellier, France

\begin{abstract}
Résumé. - L'amplification électroacoustique du bruit thermique dans le sulfure de cadmium semi-conducteur est responsable des phénomènes de saturation du courant liés à l'existence d'un domaine de haute résistivité.

L'analyse spectrale de ce dernier par diffraction Brillouin permet de connaître les bandes de fréquence amplifiées et de déterminer les variations du coefficient d'amplification pour chacune d'entre elles. On peut en outre interpréter les changements de polarisation de la lumière diffractée ainsi que le glissement en fréquence lié à la variation de conductivité au cours de la propagation du domaine.
\end{abstract}

\begin{abstract}
The acoustoelectric amplification of the thermal background in semiconducting cadmium sulfide is responsible of the current saturation when a high resistivity domain occurs.

The spectral analysis of this domain by Brillouin diffraction may allow us : to measure the acoustic gains for every frequency, to interprete the polarization change of the scattering light, to correlate the frequency shifting to the conductivity variations.
\end{abstract}

Les techniques de diffraction ont permis d'étudier l'évolution des ondes acoustiques transverses en régime de saturation du courant pour des fréquences comprises entre $250 \mathrm{MHz}$ et $4,5 \mathrm{GHz}$ dans les monocristaux de sulfure de cadmium semi-conducteur. Cette évolution se traduit essentiellement par un glissement ou reconversion vers les basses fréquences [1], [2], [3], [4] et a été interprétée qualitativement [5][6]-[7]-[8] par interactions non linéaires.

D'après B. W. Hakki et R. W. Dixon [9], ce mécanisme reste secondaire et la théorie de D. L. White [10] rend compte des glissements en fréquence observés dans le domaine, par le piégeage des porteurs libres au moment de la saturation du courant [16]. Dans cet article, nous allons également montrer que cette théorie rend compte des gains observés.

TeChniques eXPérimentales [11]. - Préparation des échantillons. - Ce sont des monocristaux de forme parallélépipédique de dimensions $4 \times 2$, $6 \times 1,6 \mathrm{~mm}^{3}$ de résistivité comprise entre 2 et $5 \Omega . \mathrm{cm}$ munis de contacts ohmiques par évaporation et diffusion d'indium, de façon à amplifier les modes acoustiques tranverses polarisés parallèlement à l'axe $C$.

Appareillage. - La figure 1 donne une vue d'ensemble du montage mécanique. La lumière incidente fournie par un laser He-Ne $2 \mathrm{~mW}$. Spectra Physics traverse un nicol polariseur avant de pénétrer dans un collimateur, l'ensemble est solidaire d'une platine goniométrique Bouty.

Le diamètre du spot lumineux atteint $150 \mu$ au niveau de l'échantillon de CdS soumis à un champ électrique pulsé de $3 \mu$ s de durée et de faible récur-

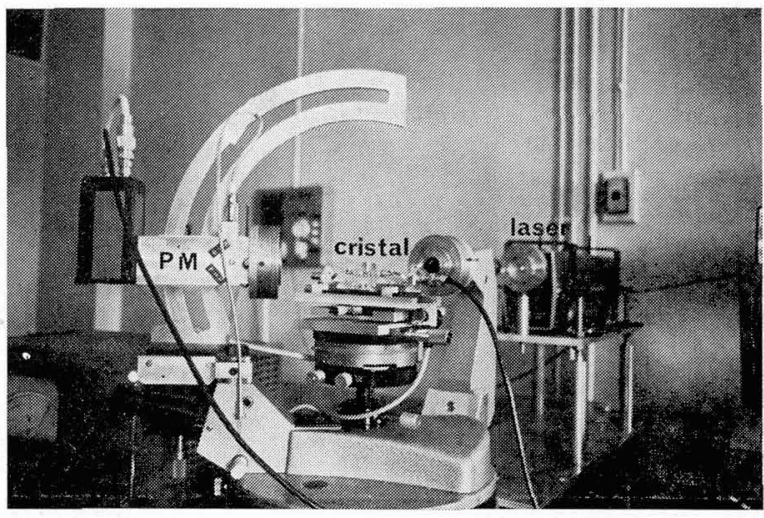

FIG. 1. - Vue d'ensemble du montage mécanique.

rence $(1 \mathrm{~Hz})$. Le photomultiplicateur de détection type RTC XP 1117 est fixé à la place de la lunette de visée et délivre un signal, qui, après passage dans un détecteur de crête, est enregistré sur une table traçante XY type Hewlett-Packard.

Afin de permettre une exploration complète du domaine, l'échantillon peut être déplacé selon les mouvements suivants :

- un mouvement angulaire horizontal pour sélectionner la fréquence acoustique à étudier ainsi que les modes hors d'axe se trouvant dans un plan horizontal,

- un premier mouvement linéaire horizontal dans le sens du champ électrique de façon à suivre l'évolution de l'amplification acoustoélectrique,

- un deuxième mouvement linéaire horizontal pour effectuer le centrage de l'échantillon par rapport à l'axe de la tête tournante, 
- enfin, un mouvement vertical nécessaire à tester l'homogénéité du domaine dans un plan perpendiculaire à la direction du champ électrique.

RÉSUltatS ET DISCUSSION. - Mesure de l'anisotropie optique. - Le sulfure de cadmium étant un cristal anisotrope, la rotation du vecteur d'onde de la lumière incidente due à la diffraction Brillouin des ondes transverses polarisées selon l'axe $C$ entraîne un changement de la vitesse de phase de l'onde lumineuse. Les règles habituelles de conservation de l'énergie et de la quantité de mouvement fournissent, par des considérations trigonométriques simples (Fig. 2A et $\mathrm{B}$ ), les angles de diffraction à l'intérieur et à l'extérieur du cristal :

$$
\begin{aligned}
& \sin \theta_{\mathrm{i}}=\frac{\hat{\lambda}_{0}}{2 n_{0} v_{\mathrm{s}}}\left(f+\frac{v_{\mathrm{s}}^{2}}{f \lambda_{0}^{2}}\left(n_{\mathrm{e}}^{2}-n_{0}^{2}\right)\right) \\
& \sin \theta_{\mathrm{s}}=\frac{\hat{\lambda}_{0}}{2 n_{\mathrm{e}} v_{\mathrm{s}}}\left(f-\frac{v_{\mathrm{s}}^{2}}{f \lambda_{0}^{2}}\left(n_{\mathrm{e}}^{2}-n_{0}^{2}\right)\right) .
\end{aligned}
$$

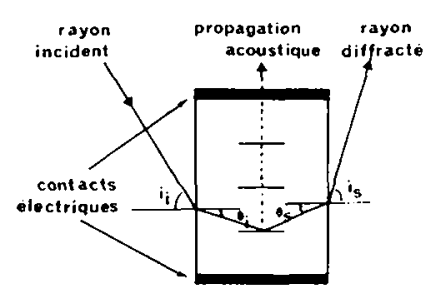

(A)

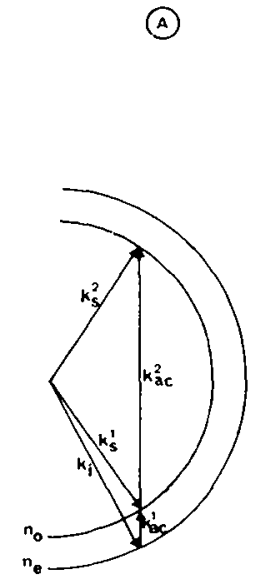

(c)

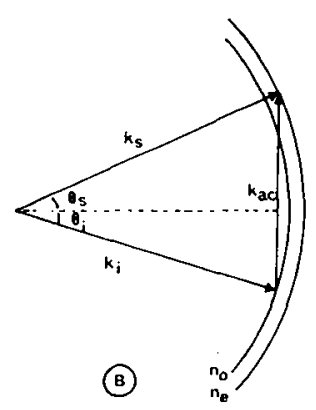

(B)

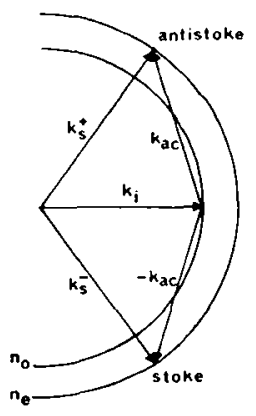

(2)
Fig. 2. - A) Processus de la diffraction dans le cas présentement étudié. B) Composition des vecteurs d'onde. C) Double diffraction en polarisation parallèle à l'axe $C$ pour une incidence fixe. D) Mise en évidence des raies stoke et antistoke correspondant à une même fréquence acoustique.

$\lambda_{0}$ étant la longueur d'onde optique dans le vide, $n_{\mathrm{e}}$ et $n_{0}$ les indices extraordinaires et ordinaires du cristal, $v_{\mathrm{s}}$ la vitesse des ondes acoustiques, $f$ la fréquence.

Pour un mode transverse se propageant dans la direction du champ électrique, il y a réversibilité des angles $i_{\mathrm{i}}$ et $i_{\mathrm{s}}$ à l'extérieur du cristal après rotation de $\pi / 2$ du vecteur d'onde de la lumière incidente. Cette propriété ne s'applique pas [11] pour les phonons hors d'axe, ce qui permet de déterminer leur direction par rapport à celle du champ électrique. La fréquence observée se déduit de $\theta_{\mathrm{i}}$ et $\theta_{\mathrm{s}}$ (relation (1) $+(2)$ ) ainsi que l'anistotropie (relation (1) - (2))

$$
\begin{gathered}
f=\frac{v_{\mathrm{s}}}{\lambda_{0}}\left(\sin i_{\mathrm{i}}+\sin i_{\mathrm{s}}\right) \\
n_{\mathrm{c}}^{2}-n_{0}^{2} \simeq 2 n \Delta n=\frac{v_{\mathrm{s}}}{\lambda_{0}}\left(\sin i_{\mathrm{i}}-\sin i_{\mathrm{s}}\right) .
\end{gathered}
$$

La valeur moyenne de $\Delta n$ obtenue est de $1,9 \times 10^{-2}$ en bon accord avec les mesures faites par interférométrie.

Mentionnons qu'avec des cristaux anisotropes, il existe (Fig. 2C), pour un angle d'incidence fixe et en polarisation parallèle à l'axe $\mathrm{C}$, deux maxima de diffraction correspondant à deux fréquences différentes. L'observation de ce phénomène, représenté figure 3, est toutefois assez délicate. En effet,

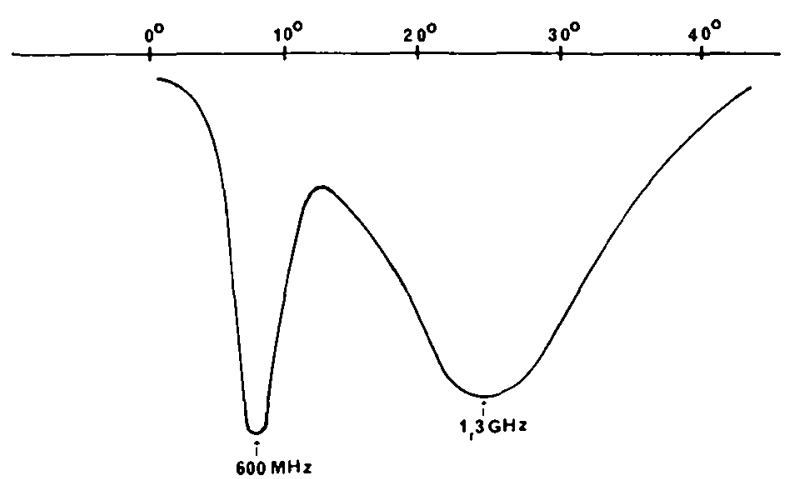

FIG. 3. - Mise en évidence expérimentale de la double difiraction par déplacement du photomultiplicateur pour une incidence fixe.

il est indispensable pour séparer les deux pics, que d'une part, les fréquences détectées $f_{1}$ et $f_{2}$ ne soient pas trop proches, et que d'autre part, leurs intensités respectives soient du même ordre de grandeur. Dans nos expériences, les deux fréquences détectées se situent entre 500 et $600 \mathrm{MHz}$ pour les basses fréquences, et entre 1,2 et $1,5 \mathrm{GHz}$ pour les hautes fréquences, en bon accord avec la relation

$$
f_{1} f_{2}=8,2 \times 10^{17}
$$

déduite des relations (1) et (2).

RAIES STOKE ET ANTISTOKE. [11]. - L'interaction de la lumière avec des phonons de même fréquence donne lieu, en toute généralité à une double diffraction en accord avec les règles de sélection

$$
\mathbf{k}_{\mathrm{s}}^{+}=\mathbf{k}_{\mathrm{i}}+\mathbf{k}_{\mathrm{ac}}
$$


absorption d'un phonon: raie antistoke

$$
\mathbf{k}_{\mathrm{s}}^{-}=\mathbf{k}_{\mathrm{i}}-\mathbf{k}_{\mathrm{ac}}^{\prime}
$$

émission d'un phonon : raie stoke.

Les vecteurs $\mathbf{k}_{\mathrm{ac}}$ et $\mathbf{k}_{\mathrm{ac}}^{\prime}$ sont égaux en module en négligeant l'anisotropie acoustique, mais différents en direction (Fig. 2D).

Remarquons que, du fait de l'anisotropie optique naturelle du $\mathrm{CdS}$, il nous est possible de détecter en incidence normale des phonons transverses se propageant dans la direction du champ électrique. Dans ce cas les vecteurs $\mathbf{k}_{\mathrm{ac}}$ et $\mathbf{k}_{\mathrm{ac}}^{\prime}$ ont même direction, c'est-à-dire que les raies stoke et antistoke sont symétriques par rapport au faisceau direct et d'égale amplitude. Cette égalité d'amplitude est due à l'équilibre statistique entre le nombre de phonons émis et absorbés dans une direction donnée.

GlisSEMENT EN FRÉQUeNCE. - La gamme de fréquences acoustiques observables par effet Brillouin dans le CdS avec un laser $\mathrm{He}-\mathrm{Ne}$ s'étend entre 0,12 et $5,5 \mathrm{GHz}$ environ. La limitation vers les hautes fréquences est régie par la relation $\sin 0=\lambda_{\mathrm{op}} / \lambda_{\mathrm{ac}}=1$, tandis que la limitation vers les basses fréquences est due à l'anisotropie naturelle du cristal (relations (1) et (2)).

Le maximum d'amplification obtenu en régime linéaire pour des échantillons de résistivité de 2 à $5 \Omega . \mathrm{cm}$ est compris entre 5 et $10 \mathrm{GHz}$. Seul, le comportement des fréquences inférieures peut être étudié. Les échantillons sont sélectionnés avant toute étude par effet Brillouin et seuls sont retenus ceux qui possèdent un palier de saturation très plat afin d'éviter la présence d'inhomogénéités. Aucune diffraction n'est détectée dans la partie ohmique du courant, les premiers signaux apparaissent dès la saturation. Il n'est cependant pas possible de faire des mesures de gain, vu l'étroitesse de cette région $(\simeq 100 \mu)$ comparée à la largeur du faisceau laser. Sur la figure 4, sont représentées les variations du signal du photomultiplicateur en échelle logarithmique en fonction de la position du spot laser sur le cristal et ce, pour différentes fréquences. Les signaux les plus importants se situent entre $500 \mathrm{MHz}$ et $1 \mathrm{GHz}$. Les gains nets déduits des courbes précédentes atteignent jusqu'à $480 \mathrm{~dB} / \mathrm{cm}$ dès la chute du courant pour des fréquences voisines de $1 \mathrm{GHz}$ puis, accusent une très nette diminution aux hautes fréquences, lors de la propagation du domaine. Ce phénomène s'explique par le piégeage des porteurs par les ondes acoustiques entraînant ainsi une diminution du nombre de porteurs libres qui participent à l'amplification acoustoélectrique [16]. La fréquence correspondant au gain maximum donné par la formule de D. $L$. White, se déplace donc vers les basses fréquences puisque $\omega_{c}$ diminue. Il y a donc un processus de reconversion vers les basses fréquences d'autant plus marqué que les variations de conductivité dans le domaine sont plus importantes. Les champs élec-

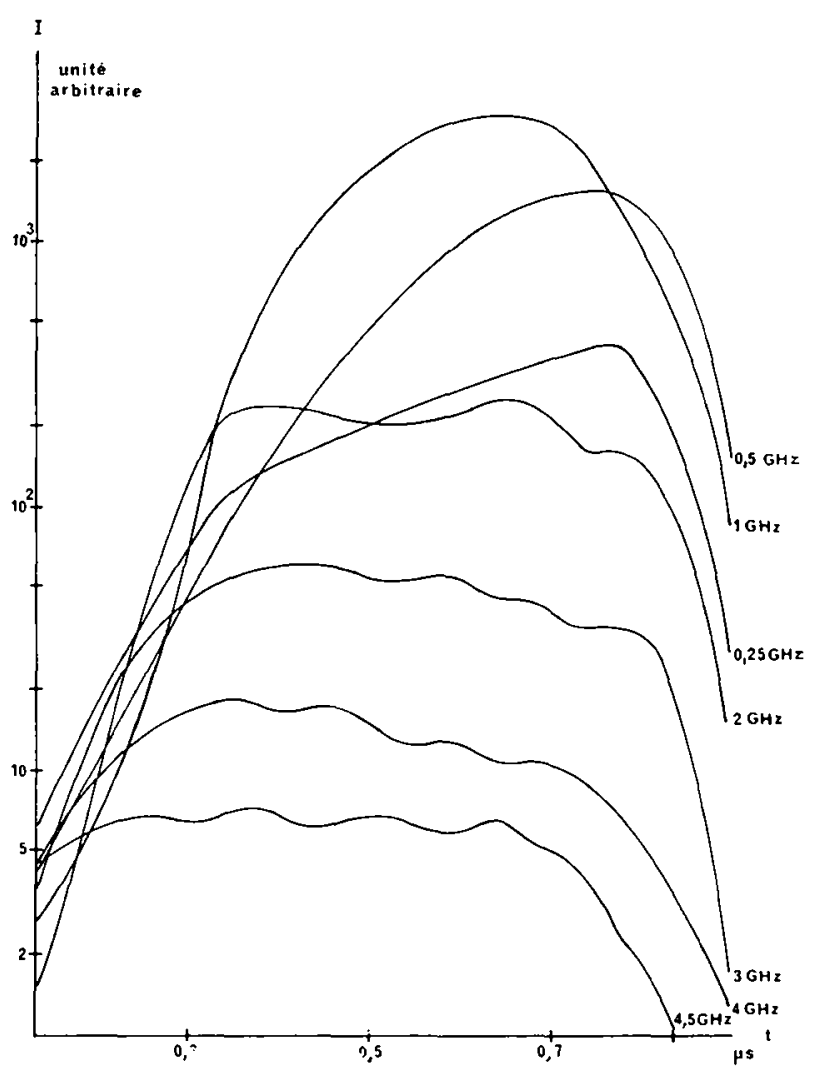

FIG. 4. - Variations de l'intensité lumineuse diffractée en fonction du temps à partir de la fin du courant ohmique, pour différentes fréquences.

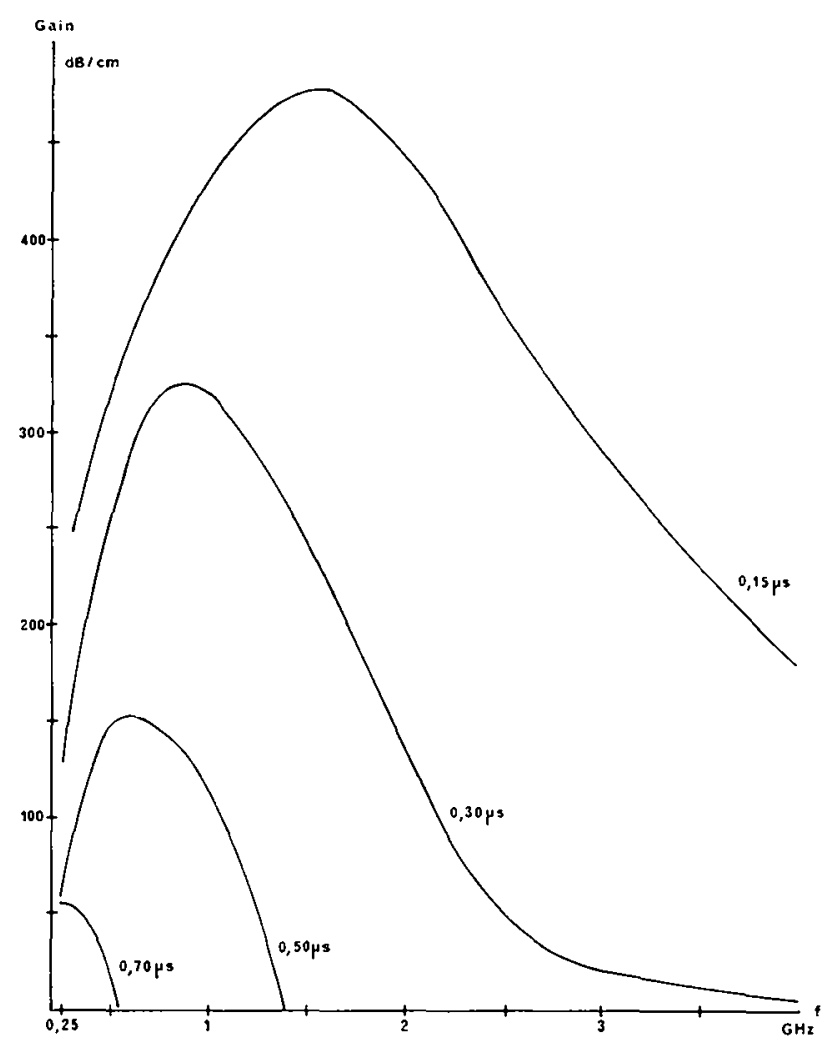

FIG. 5. - Variations du gain net déduit des courbes précédentes en fonction de la fréquence pour différents temps d'échantillonnage à partir de la fin du courant ohmique. 
triques mesurés par des sondes en tungstène lors du passage du domaine avoisinent $10^{4} \mathrm{~V} / \mathrm{cm}$, ce qui nous permet, connaissant la fréquence la plus amplifiée en chaque point du cristal, c'est-à-dire la conductivité, de calculer le coefficient d'amplification pour différentes valeurs de $\omega_{\mathfrak{c}}$

$$
\alpha=-\frac{K^{2}}{2 v_{\mathrm{s}}} \cdot \omega_{\mathrm{c}} \frac{\gamma}{\gamma^{2}+\left(\frac{\omega_{\mathrm{c}}}{\omega}+\frac{\omega}{\omega_{\mathrm{d}}}\right)^{2}}
$$

avec les notations habituelles.

$$
\begin{gathered}
\gamma \text { étant très supérieur à } \frac{\omega_{\mathrm{c}}}{\omega}+\frac{\omega}{\omega_{\mathrm{d}}} \text { on en déduit : } \\
\alpha=-\frac{K^{2}}{2 v_{\mathrm{s}}} \frac{\omega_{\mathrm{c}}}{\gamma} .
\end{gathered}
$$

Ce gain linéaire en $\omega_{\varepsilon}$ est indépendant de la fréquence dans une large gamme autour de $\sqrt{\omega_{c}} \overline{\omega_{d}}$, ce qui est en accord avec les résultats expérimentaux représentés figure 6 , aux pertes acoustiques près.

Les variations de conductivité en fonction de l'abscisse dans le cristal sont dans un rapport 100 environ entre le régime ohmique et le début du palier, elles atteignent un facteur $10^{3}$ à la fin du palier, $\omega_{\mathfrak{c}}$ devient alors sensiblement inférieur à $\omega_{d}$ et nous assistons à un dépiégeage des porteurs provoquant la destruction du domaine.

EFFETS NON LINÉAIRES ET DIFFRACTIONS MULTIPLES. - On remarque, sur les courbes de la figure 4, l'apparition de maxima secondaires de diffraction aux hautes fréquences. Deux interprétations qualitatives peuvent en être données:

- la première, suggérée par D. L. Spears [13] lors de son étude des domaines sur GaAs, consiste à faire intervenir les diffractions multiples. Il est en effet possible, étant calé sur une fréquence $f_{1}\left(i_{\mathbf{i}}\right.$ et $i_{\mathrm{s}}$ fixés), de détecter les ordres $n$ de diffraction impairs supérieurs des fréquences $f_{2}=f_{1} / n$, ces dernières ayant chacune, du fait de la reconversion vers les basses fréquences, une amplitude bien plus importante que celle de la fréquence $f_{1}$;

- une seconde interprétation peut être donnée

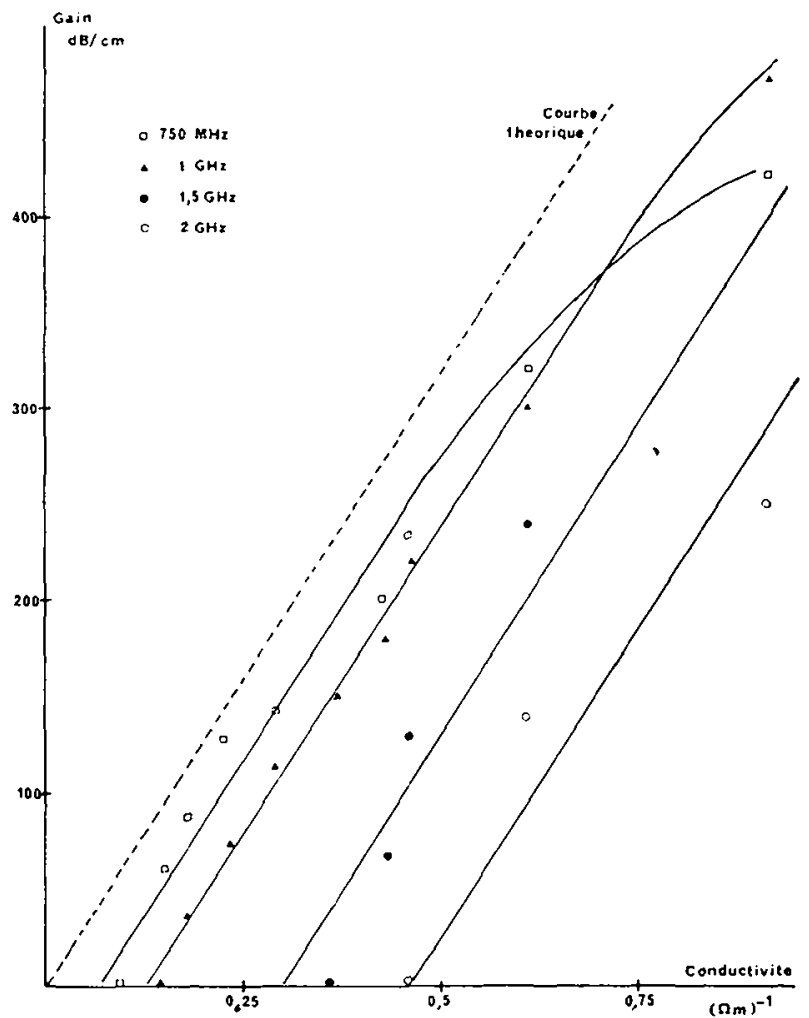

FIG. 6. - Variations du gain en fonction de la conductivité pour différentes fréquences. La courbe théorique est tracée pour une valeur du champ électrique de $10^{4} \mathrm{~V} / \mathrm{cm}$.

en faisant intervenir les effets non linéaires, car la présence au même endroit de deux fréquences $f_{1}$ et $f_{2}$ engendre les fréquences somme $f_{1}+f_{2}$ et différence $f_{1}-f_{2}$. Le calcul de ces termes du $2^{e}$ ordre a été effectué par E. M. Conwell et A. K. Canguly [14] et, la preuve expérimentale de leur existence dans le CdS photoconducteur vient d'être établie par G. Sagnes, C. Roustan et M. Rouzeyre [15] en faisant interagir deux ondes de surface à la fréquence $\omega$ et en détectant le signal à $2 \omega$.

Quoi qu'il en soit, ces phénomènes n'affectent que très peu l'amplitude du signal fondamental pour lequel le gain est positif.

\section{Bibliographie}

[1] ZuCKer J. and ZEMON S., Appl. Phys. Lett. 9 (1966) 398, erratum : Appl. Phys. Lett. 10 (1967) 212.

[2] ZuCKer J., Zemon S. and WaSko J. H., II-VI Semiconducting Compounds 1967, International Conference, D. G. Thomas Ed. (Benjamin New York 1967), 919-927.

[3] Zucker J., Zemon S., Conwell E. M. and Canguly A. K., International Conference on the Light Scattering Spectra of Solids, 1968, G. B. Wright Ed. (Springer, New York 1969), 615-621.

[4] Zucker J., Zemon S. and Wasko J. H., Ninth International Conference on the Physics of Semiconductors, Moscow 1968 (Nanka, Leningrad 1968), 2, 904-910.

[5] Zucker J. and Zemon S., J. Acoust. Soc. Am. 49 (1971) 1038.
[6] Wettlung W., II-VI Semiconducting Compounds 1967 International Conference D. G. Thomas Ed. (Benjamin New York 1967), 928-935.

[7] Ishida A. and Inuishi Y., Phys. Lett. 27A (1968) 442.

[8] Ishida A. and Inuishi Y., J. Phys. Soc. Jap. 27 (1969) 911.

[9] Hakki B. W. and Dixon R. W., Appl. Phys. Lett. 14 (1969) 185.

[10] White D. L., J. Appl. Phys. 33 (1962) 2547.

[11] GiRaRD P., Thèse Montpellier (1972).

[12] BENEDeK G. B. and FRITSCH K., Phys. Rev 149 (1966) 647.

[13] Spears D. L., Phys. Rev. B 2 (1970) 1931

[14] Conwell E. M. and Canguly A. K., Phys. Rev. B 4 (1971) 2535

[15] Sagnes G., Roustan C. and Rouzeyre M. (à paraître).

[16] Lepetre T., Suppl. J. Physique 33 (1972) C 6. 